\title{
A High-Capacity Image Steganography Method Using Chaotic Particle Swarm Optimization
}

\author{
Aya Jaradat $(\mathbb{D}$, Eyad Taqieddin $(\mathbb{D}$, and Moad Mowafi \\ Department of Network Engineering and Security, Jordan University of Science and Technology, Irbid, Jordan \\ Correspondence should be addressed to Eyad Taqieddin; eyadtaq@just.edu.jo
}

Received 20 November 2020; Revised 21 March 2021; Accepted 31 May 2021; Published 8 June 2021

Academic Editor: Jinwei Wang

Copyright (C) 2021 Aya Jaradat et al. This is an open access article distributed under the Creative Commons Attribution License, which permits unrestricted use, distribution, and reproduction in any medium, provided the original work is properly cited.

\begin{abstract}
Image steganography has been widely adopted to protect confidential data. Researchers have been seeking to improve the steganographic techniques in order to increase the embedding capacity while preserving the stego-image quality. In this paper, we propose a steganography method using particle swarm optimization and chaos theory aiming at finding the best pixel locations in the cover image to hide the secret data while maintaining the quality of the resultant stego-image. To enhance the embedding capacity, the host and secret images are divided into blocks and each block stores an appropriate amount of secret bits. Experimental results show that the proposed scheme outperforms existing methods in terms of the PSNR and SSIM image quality metrics.
\end{abstract}

\section{Introduction}

Information security has become a major topic due to its important role in data communication. It offers techniques to secure the information by avoiding any illegal access, change, damage, or disclosure of it [1]. The impact and importance of information security in daily life applications are supported by two ways, cryptography and steganography $[2,3]$, where steganography maintains the data format preserved [4]; on the other hand, cryptography converts the data into difficult to read codes [5].

Steganography is a Greek word that comes from the word Steganos; it appeared around 2,500 years ago [6] and has been used to embed information in different media such as manuscripts, videos, images, and audio. It provides a way to communicate safely so that it does not attract suspicion and attention during data transmission. It goes hand-inhand with the accompanying progress in the area of digital communications, which is clearly evident in the growth of data and users.

The efficiency of any steganographic method greatly depends on the selection of the embedding area in the cover medium intelligently and accurately [7]. This can affect the embedding capacity and the quality of the cover medium. In this work, we propose a new method for image hiding to improve the efficiency of embedding without compromising the quality of the resulting stego-image. The proposed method utilizes the particle swarm optimization and chaos theory to implement the embedding process.

Particle swarm optimization (PSO) is an efficient and easy-to-implement algorithm. It is closely linked to artificial life systems and works in a collective and cooperative manner [8]. The PSO algorithm does not require an enormous number of control parameters and can be easily accomplished through balanced computing as it is applied to gathering of particles. PSO focuses mainly on a number of random particles and aims to find the optimal solution through repetition [9]. It has been used in various fields, including steganography. For example, in $[10,11]$, PSO was used to find the optimal pixel in the cover image to embed the secret data.

PSO depends on finding the best position for an ideal particle location and the best global particle location. However, one drawback to using it is that the particles become susceptible to premature convergence, which leads to trapping the swarm in an ideal local area and the inability to discover any new area located in the local optimal solution. Thus, access to the global optimal solution becomes relatively weak. 
In this work, our goal is to avoid this problem by employing the chaos theory [12], which depends mainly on random movement to generate random sequences that help in preventing any repetitions in the search area. Chaos theory can improve the quality of search by determining the location more accurately and achieves a balance between the global and local position search capabilities.

The rest of this paper is organized as follows: Section 2 reviews the related work and Section 3 presents background on PSO and chaotic map. Section 4 describes the proposed method and Section 5 discusses the experimental setup and evaluation of the results. Section 6 concludes the paper and provides future work.

\section{Related Work}

In [13], the authors proposed a technique that relies on the Vernam Cipher algorithm and the false random key and adopts the least significant bit (LSB) steganography method in order to hide the secret image bits into the cover image. In [14], a new scheme based on pixel-value differencing and PSO was proposed to hide confidential information in the cover image.

The authors in [15] used the principle of pixel overlapping to hide a large amount of information with high image quality. In [16], a quantum information hiding protocol was suggested based on image expansion using the quantum log-polar image representation and the Grover search algorithm to extract the precisely designed secret message and determine the correct quantitative image copy. In [17], the genetic algorithm (GA) was used with a linear convergence generator to hide confidential data into the cover image by specifying the appropriate locations to include at least two bits in each pixel. The proposed model provided high cloaking for the data but low embedding capacity due to the inclusion of only two bits.

The work in [18] provided a technique depending on GA and LSB substitution to increase the embedding capacity and reduce the distortion that may affect the resultant image.

In [19], a technique using the Knight Tour algorithm with LSB is proposed. It aims to shorthand the image and strengthen the security of the stego-image. The Chi-square statistical attack was applied to evaluate the security level of the proposed method. The authors in [20] relied on differencing and substitution mechanisms in the process of concealing high-capacity information. First, LSB substitution is applied to the two lower significant bits, then the quotient value differencing (QVD) is applied to the remaining six bits.

An embedding method was introduced in [21] based on the use of PSO for optimal selection of pixel and wavelet transformation with the aim of masking a secret sound signal in the cover image. In [22], the authors proposed a hybrid information masking scheme that consists of a mixture of noise visibility function and an optimal coding scheme to achieve an increase in the payload capacity. Furthermore, a steganography procedure was proposed in [23] depending on the PSO algorithm with the aim of achieving consistent compatibility with the size of the secret data so as to accomplish a perfect stego-image quality through the substitution matrix intended to transform the secret data in the cover image.

The authors in [24] presented a technique that depends on GA to embed the secret data bits into the cover images. A simple LSB is applied first; then, GA is used to change the pixel values to make the stego-image safe against RS attacks. In [25], another method that depends on GA was presented. It divides the cover images into parts, then the best pixels are found in each part to complete the embedding process.

In this paper, we study applying the PSO algorithm with chaotic map in steganography aiming at improving the quality of the stego-image as well as the embedding capacity.

\section{Background}

3.1. Standard PSO Algorithm. The PSO algorithm aims to work in a collective and cooperative manner in a smart way. It appeared in 1995 and witnessed a number of enhancements in many areas, as it is closely linked to the evolutionary algorithms and artificial life [8]. It has many advantages including the following: (a) it does not require a large number of control parameters, so it is anything but not difficult to execute; (b) it can be accomplished easily through balanced computing as it is applied to gathering of particles. It focuses mainly on a number of random particles and aims to find the optimal solution through repetition $[9,26]$.

The PSO algorithm depends mainly on the population as it consists of a group of individuals or particles called swarms, and these particles move to the search area with multiple dimensions to start searching for a solution, similar to searching for food. The individuals depend in their directions on the sociopsychological trends of other particles, which leads to a change in particle conditions as a result of the change in speed. These changes lead to the movement of the particles toward the best position in the search area which is known as the best global position (gbest), and each movement of the particle through its favorable position is known as the best particle position (pbest). These two activities direct the particles to move toward the ideal solution [11]. The new location $x_{i}^{t+1}$ and velocity $v_{i}^{t+1}$ of particle $i$ at iteration $t+1$ can be expressed as

$$
\begin{aligned}
v_{i}^{t+1}= & w \times v_{i}^{t}+c_{1} \times r_{1} \times\left(\text { pbest }_{i}^{t}-x_{i}^{t}\right) \\
& +c_{2} \times r_{2} \times\left(\text { gbest }^{t}-x_{i}^{t}\right), \\
x_{i}^{t+1}= & x_{i}^{t}+v_{i}^{t+1},
\end{aligned}
$$

where $r_{1}$ and $r_{2}$ are random numbers between 0 and $1, c_{1}$ and $c_{2}$ are acceleration factors, and $w$ is the inertia weight that controls the velocity.

3.2. Chaotic Maps. A chaotic map function indicates a type of development that shows chaotic and random behavior that is not predictable, but it is characterized by a type of regularity and ergodicity that works to prevent points repetition from occurring in the search ranges caused by the random sequence. The chaotic equation is also characterized 
by its sensitivity to the initial values. If a slight change occurs, it leads to major changes in the behavior of the system. Logistic map is one of the simplest forms of a chaotic system and it has been used in many fields including steganography $[27,28]$. In this work, we use the logistic map to produce primary particles distributed in a bound structure and recreate a specific number of particles. More insights on the logistic map are given in the next section.

\section{Proposed Method}

One of the disadvantages of the PSO algorithm is the premature convergence toward the optimal local solution. In this work, we propose a chaotic PSO (CPSO) algorithm to make the PSO algorithm progressively effective in the steganography process by locating the best pixel locations in the cover image to embed the secret image bits while maintaining the quality of the stego-image resulting from the embedding process.

4.1. CPSO Algorithm. In the proposed algorithm, we utilize the logistic map to generate the chaotic variables and map these variables to the search space. The logistic equation can be expressed as

$$
z_{i+1}=\mu z_{i}\left(1-z_{i}\right)
$$

where $z_{i}$ is a chaotic variable in the interval $(0,1)$ and $\mu$ is a parameter in the interval $[0,4]$ that controls the behavior of the logistic map. When $\mu$ is in the range $[3,4]$, the system behaves in a chaotic manner.

For the particles speed, we utilize a sigmoid function for accurate positioning, which can be expressed by the following equation:

$$
s\left(v_{i}^{t+1}\right)=\frac{1}{1+e^{-v_{i}^{t+1}}} .
$$

In the traditional PSO algorithm, random numbers are used to initialize the population particles. Since the initial velocity and direction of each particle in the population are irregular and not constant, this may lead to missing some spatial positions. On the other hand, using a chaotic sequence instead of the random numbers for initialization is considered a robust method that increases the diversity of the particles and enhances the performance of the PSO algorithm by preventing premature convergence. In the proposed algorithm, the logistic map is applied when the particle's speed and position are initialized, and instead of using the random numbers $r_{1}$ and $r_{2}$, we use two chaotic sequences that are created according to the following equations:

$$
\begin{cases}x_{i}^{t+1}=0, & r(t+1)<s\left(v_{i}^{t+1}\right), \\ x_{i}^{t+1}=1, & r(t+1) \geq s\left(v_{i}^{t+1}\right),\end{cases}
$$

where $r(t+1)$ is a real number generated between $[0,1]$. Accordingly, the particle velocity is calculated as follows:

$$
v_{i}^{t+1}=w \times v_{i}^{t}+c_{1} \times \text { Chaotic1 }() \times\left(\text { pbest }_{i}^{t}-x_{i}^{t}\right)+c_{2} \times \text { Chaotic2 }() \times\left(\text { gbest }^{t}-x_{i}^{t}\right),
$$

where Chaotic is a function based on the logistic map results in equation (3). Algorithm 1 shows the pseudocode of the proposed CPSO algorithm.

4.2. Scanning Order in Cover Image. In this work, we use LSB substitution to hide a secret image into the cover image. To do so, we model the steganography problem as a search problem in which the goal is to find the best pixel locations in the cover image to hide the secret image with minimum distortion. This involves scanning the pixels of the cover image and determining the order and position of the pixels for embedding the secret image. There are different orders and positions that give a different stego-image quality [10]. Figure 1 shows examples of different pixel scanning orders for an image with dimension $5 \times 5$. In this work, we use the CPSO algorithm to find the best order and position in the cover image that maximizes the quality of the stego-image.

4.3. Particles Representation. In the CPSO algorithm, nine particles are defined that correspond to the variables shown in Table 1 [10]. The direction of pixel scanning in the cover image consists of 16 possible states. X-offset and Y-offset represent the starting point position. Bit-planes specify the
LSBs in the cover image that are used for embedding as shown in Table 2 [10]. SB-pole and SB-dire specify the pole and direction of secret bits, respectively, while BP-Dire specifies the direction of the LSB planes as shown in Table 3 [10].

4.4. Data Embedding. To embed the secret message, in our case an image $S(k \times v)$, into the cover image $C(m \times n)$, the secret image is first divided into four separate blocks, and each block is converted into binary according to the SB-pole and SB-dire particles. Then, the MSB of each pixel is extracted to produce a sequence of bits with length $l$ for embedding. The cover image is then divided into four separate blocks each of length $w$. Accordingly, the number of secret bits that can be embedded in each block can be calculated as $l / w$. After that, the CPSO algorithm is applied to find the best position of the pixels in each block. Within the swarm, the number of pixels in each block that are needed to embed the secret bits should be determined as the number of pixels varies depending on the number of secret bits to be embedded. Finally, the number of pixel bits in the cover image is compared with the number of secret bits. If the number of secret bits is less than the pixel bits, the secret bits are embedded into the pixels to obtain the stego-image. 
Input: Number of particles $(N)$, maximum iterations

Output: Global best particles position (gbest)

(1) for $i=1$ to $N / /$ Initialize particles velocity and position

(2) $x_{i} \longleftarrow \operatorname{Chaotic}\left(x_{\min }, x_{\max }\right)$

(3) $\quad v_{i} \longleftarrow$ Chaotic $\left(v_{\min }, v_{\max }\right)$

(4) pbest $\longleftarrow x_{i} / /$ Initialize particles local best position

(5) gbest $\longleftarrow \phi / /$ Initialize particles global best position

(6) end for

(7) do

(8) for $i=1$ to $N$

(9) if $f\left(x_{i}\right)>f\left(\right.$ pbest $\left._{i}\right)$

(10) $\quad$ pbest $_{i} \longleftarrow x_{i} / /$ Update local best position

(11) end if

(12) if $f$ ( pbest $\left._{i}\right)>f$ (gbest)

(13) $\quad$ gbest $\longleftarrow$ pbest $_{i} / /$ Update global best position

(14) end if

(15) end for

(16) for $i=1$ to $N$

(17) Update $v_{i}$ using equation (6)

(18) Update $x_{i}$ using equation (2)

(19) end for

(20) while (Maximum iterations or stopping criterion is not attained)

Algorithm 1: Pseudocode of the proposed CPSO algorithm.

\begin{tabular}{|c|c|c|c|c|}
\hline 1 & 2 & 3 & 4 & 5 \\
\hline 6 & 7 & 8 & 9 & 10 \\
\hline 11 & 12 & 13 & 14 & 15 \\
\hline 16 & 17 & 18 & 19 & 20 \\
\hline 21 & 22 & 23 & 24 & 25 \\
\hline
\end{tabular}

(a)

\begin{tabular}{|c|c|c|c|c|}
\hline 25 & 24 & 23 & 22 & 21 \\
\hline 20 & 19 & 18 & 17 & 16 \\
\hline 15 & 14 & 13 & 12 & 11 \\
\hline 10 & 9 & 8 & 7 & 6 \\
\hline 5 & 4 & 3 & 2 & 1 \\
\hline \multicolumn{4}{|c}{} \\
\hline
\end{tabular}

(b)

\begin{tabular}{|l|l|l|l|l|}
\hline 21 & 16 & 11 & 6 & 1 \\
\hline 22 & 17 & 12 & 7 & 2 \\
\hline 23 & 18 & 13 & 8 & 3 \\
\hline 24 & 19 & 14 & 9 & 4 \\
\hline 25 & 20 & 15 & 10 & 5 \\
\hline
\end{tabular}

(c)

Figure 1: Examples of pixel scanning order.

Otherwise, the embedding process cannot be performed. Figure 2 illustrates the process of data embedding and extraction.

A common quality metric to measure the difference between the cover image and the stego-image is the peak signal to noise ratio (PSNR). The higher the PSNR value, the less difference between the cover image and stego-image. In the proposed CPSO algorithm, the objective is to maximize the value of PSNR. In Algorithm 1, we consider the PSNR value as the objective function:

$$
f\left(x_{i}\right)=\operatorname{PSNR}(C, S) .
$$

Accordingly, the pbest and gbest values are iteratively updated based on the PSNR value. pbest is updated when the PSNR value is greater than the previous one, while gbest is updated when the PSNR value is globally higher. Algorithm 2 shows the pseudocode of the data embedding process.

4.5. Data Extraction. To extract the secret image, the particles are first extracted from the last row of the stego-image. The sequence of secret bits is then extracted using the corresponding particles to obtain the secret image. Algorithm 3 shows the pseudocode of the data extraction process. 
TABle 1: Particles representation.

\begin{tabular}{lccc}
\hline Particle name & Value range & Length & Description \\
\hline Direction & $0-15$ & 4 bits & Direction of pixel scanning \\
X-offset & $0-255$ & 8 bits & X-offset of starting point \\
Y-offset & $0-255$ & 8 bits & Y-offset of starting point \\
Bit-planes & $0-15$ & 4 bits & LSBs used for secret bit insertion \\
SB-pole & $0-1$ & 1 bit & Pole of secret bits \\
SB-dire & $0-1$ & 1 bit & Direction of secret bits \\
BP-dire & $0-1$ & 1 bit & Direction of bit-planes \\
X-side-length & $0-255$ & 1 bits & Dimension of block in $x$-axis \\
Y-side-length & $0-255$ & 1 bit & Dimension of block in $y$-axis \\
\hline
\end{tabular}

TABle 2: Possible values for bit-planes particle.

\begin{tabular}{|c|c|}
\hline Value & Description \\
\hline 0000 & Use none of LSBs \\
\hline 0001 & Use $1^{\text {st }}$ LSB \\
\hline 0010 & Use $2^{\text {nd }} L S B$ \\
\hline 0011 & Use $1^{\text {st }}$ and $2^{\text {nd }}$ LSBs \\
\hline 0100 & Use $3^{\text {rd }}$ LSB \\
\hline 0101 & Use $1^{\text {st }}$ and $3^{\text {rd }}$ LSBs \\
\hline 0110 & Use $2^{\text {nd }}$ and $3^{\text {rd }}$ LSBs \\
\hline 0111 & Use $1^{\text {st }}, 2^{\text {nd }}$, and $3^{\text {rd }}$ LSBs \\
\hline 1000 & Use $4^{\text {th }}$ LSB \\
\hline 1001 & Use $1^{\text {st }}$ and $4^{\text {th }}$ LSBs \\
\hline 1010 & Use $2^{\text {nd }}$ and $4^{\text {th }}$ LSBs \\
\hline 1011 & Use $1^{\text {st }}, 2^{\text {nd }}$, and $4^{\text {th }}$ LSBs \\
\hline 1100 & Use $3^{\text {rd }}$ and $4^{\text {th }}$ LSBs \\
\hline 1101 & Use $1^{\text {st }}, 3^{\text {rd }}$, and $4^{\text {th }}$ LSBs \\
\hline 1110 & Use $2^{\text {nd }}, 3^{\text {rd }}$, and $4^{\text {th }}$ LSBs \\
\hline 1111 & Use four LSBs \\
\hline
\end{tabular}

TABle 3: Possible values for SB-pole, SB-dire, and Bp-dire particle.

\begin{tabular}{|c|c|c|}
\hline Particle name & Value & Description \\
\hline \multirow{2}{*}{ SB-pole } & 0 & No change is made to secret bits \\
\hline & 1 & All secret bits are complemented \\
\hline \multirow{2}{*}{ SB-dire } & 0 & No change is made to secret bits \\
\hline & 1 & Secret bits are reversed from end to beginning \\
\hline \multirow{2}{*}{ BP-dire } & 0 & Bit-planes are used from MSB to LSB \\
\hline & 1 & Bit-planes are used from LSB to MSB \\
\hline
\end{tabular}

\section{Experimental Results}

In this section, we evaluate the performance of the proposed CPSO-based steganography method and compare it against existing PSO-based methods. We consider two parameters for evaluation: the embedding capacity and the quality of the stego-image. The capacity is measured by the number of secret bits that are embedded in the cover image. The quality of the stego-image can be measured by different metrics such as PSNR and structural similarity index (SSIM).

PSNR measures the average pixel difference between the cover image and the stego-image [29]. The higher the value of PSNR, the better the quality of the stego-image. The value of PSNR is calculated based on the value of the mean square error (MSE), which represents the value of the squared error between the stego-image and the cover image. The smaller the value of MSE, the lower the error. PSNR and MSE can be calculated as follows:

$$
\mathrm{MSE}=\frac{1}{W \times H} \sum_{i=1}^{W} \sum_{j=1}^{H}\left(x_{i j}-y_{i j}\right)^{2}, \mathrm{PSNR}=10 \log \frac{255^{2}}{\mathrm{MSE}}
$$

where $W \times H$ is the resolution of the cover image and $x_{i j}$ and $y_{i j}$ indicate the value of each pixel in the cover and stego-images.

SSIM is a predictor of the perceived quality of images and videos. It realizes image deterioration through the perceived change in the structural image data [30]. The value 


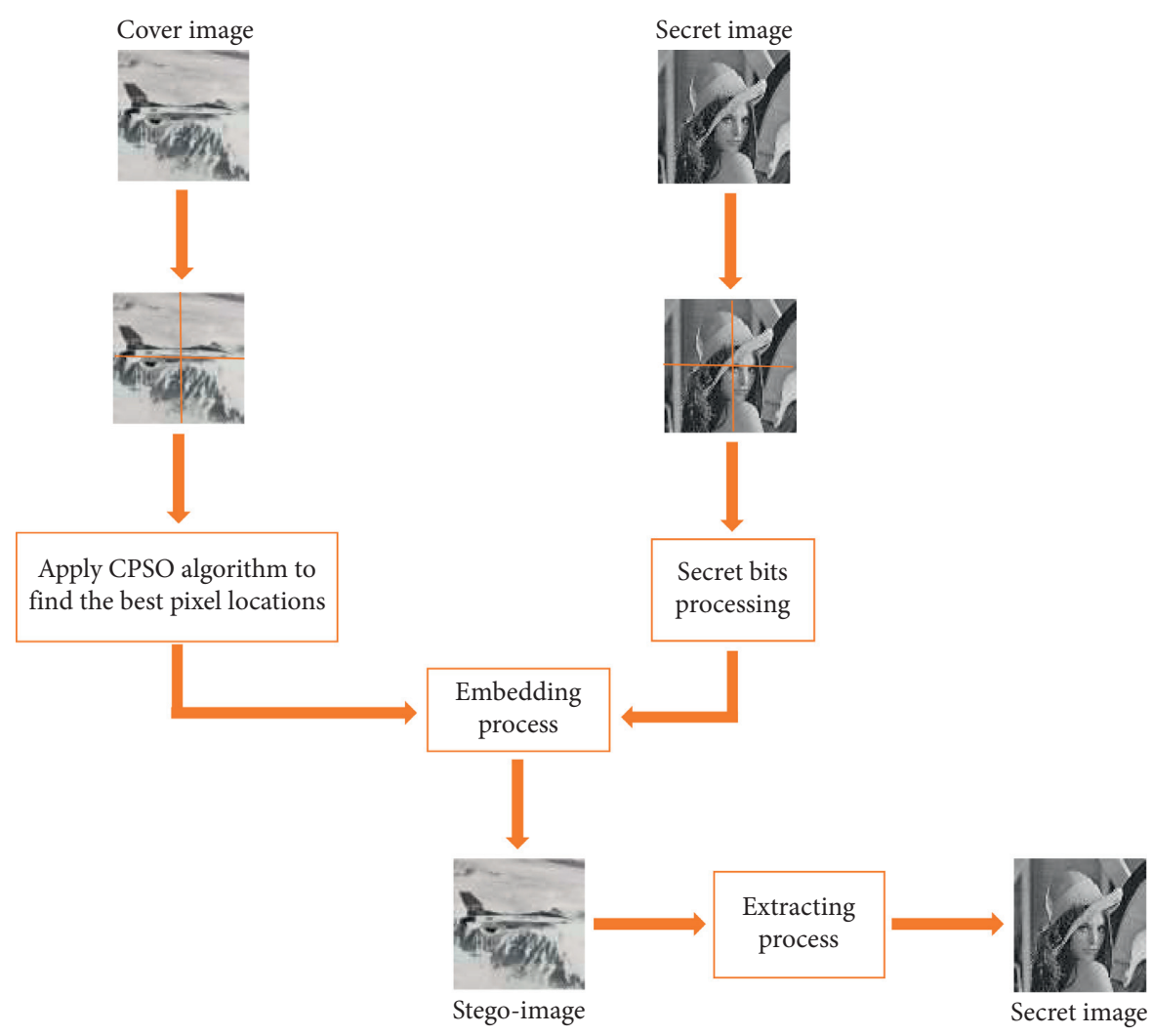

Figure 2: Data embedding and extraction.

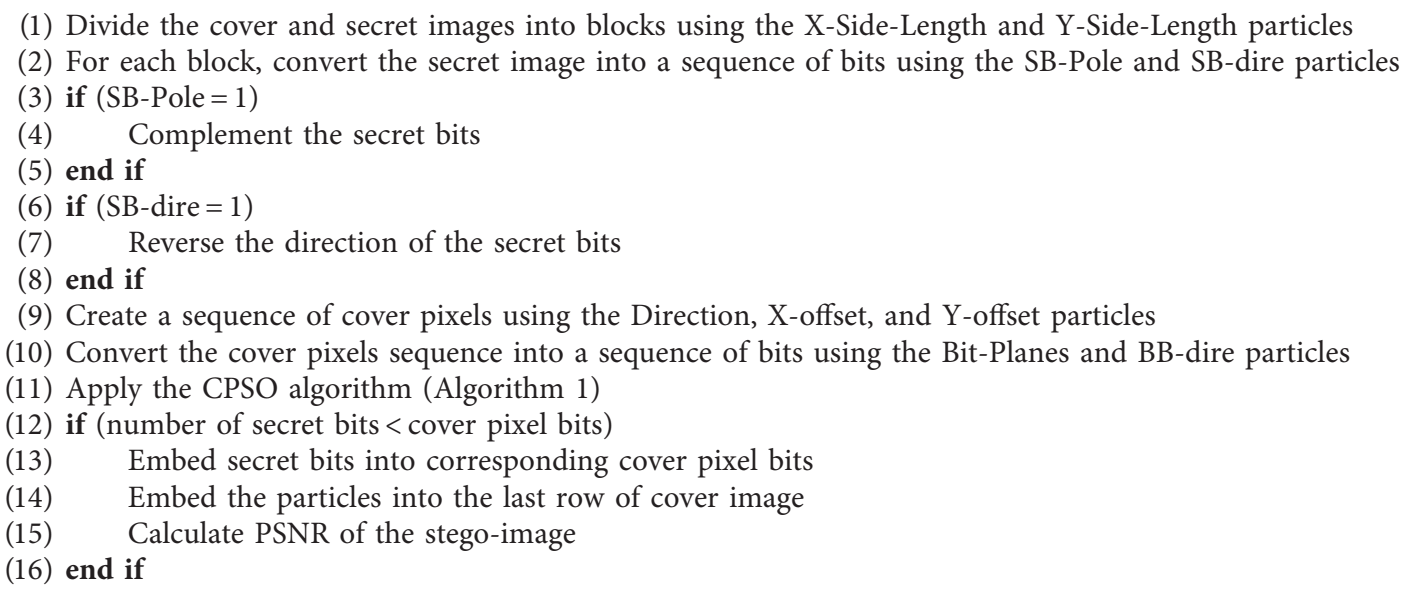

Algorithm 2: Pseudocode of data embedding.

of SSIM is in the range $[0,1]$, where the value of 1 indicates the highest quality. SSIM can be calculated between two image windows $x$ and $y$ of common size as

$$
\operatorname{SSIM}=\frac{\left(2 \mu_{x} \mu_{y}+c_{1}\right)\left(2 \sigma_{x y}+c_{2}\right)}{\left(\mu_{x}^{2}+\mu_{y}^{2}+c_{1}\right)\left(\sigma_{x}^{2}+\sigma_{y}^{2}+c_{2}\right)},
$$

where $\mu$ and $\sigma$ are the mean and variance, respectively, and $c_{1}$ and $c_{2}$ are two constants included to avoid instability.

In the experiments, secret images with various resolutions are embedded into the cover image. Figure 3 shows the five standard images used as cover and secret images. First, the cover and secret images are divided into four separate blocks. Then, for each block, the CPSO algorithm is applied 
(1) Extract the particles from the last row of stego-image

(2) Prepare the image blocks using the X-Side-Length and Y-Side Length particles

(3) Create a sequence of stego pixels using the Directions, X-offset, and Y-offset particles

(4) Convert the stego pixels sequence into a sequence of bits using the Bit-Planes and BB-dire particles

(5) if $(\mathrm{SB}-\mathrm{Pole}=1)$

(6) Complement the secret bits

(7) end if

(8) if (SB-dire $=1)$

(9) Reverse the direction of the secret bits

(10) end if

(11) Extract the secret pixels from the secret bits

(12) Render secret image using the secret pixels

(13) end

Algorithm 3: Pseudocode of data extraction.

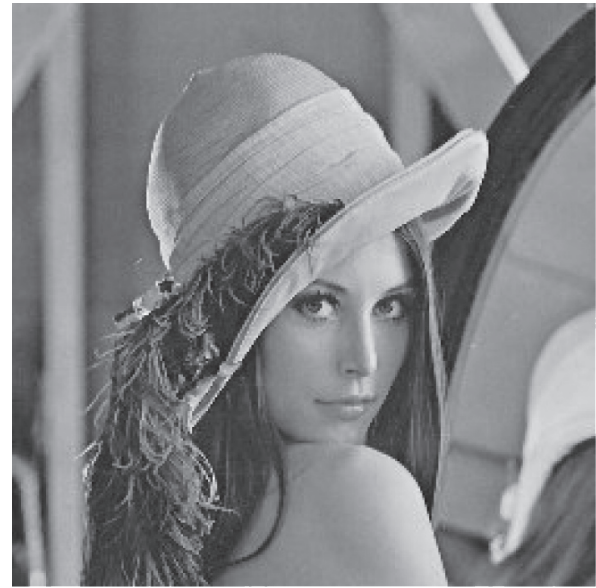

(a)

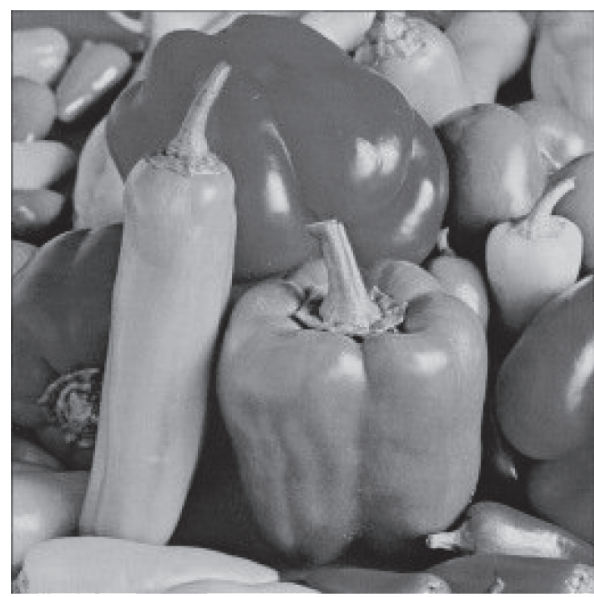

(c)

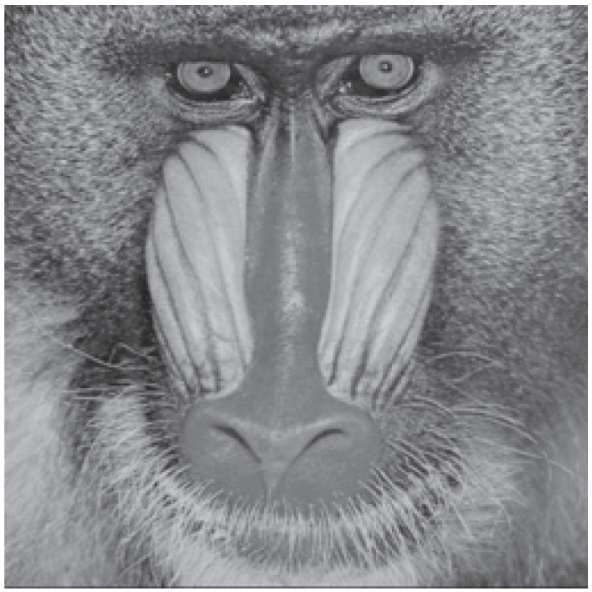

(b)

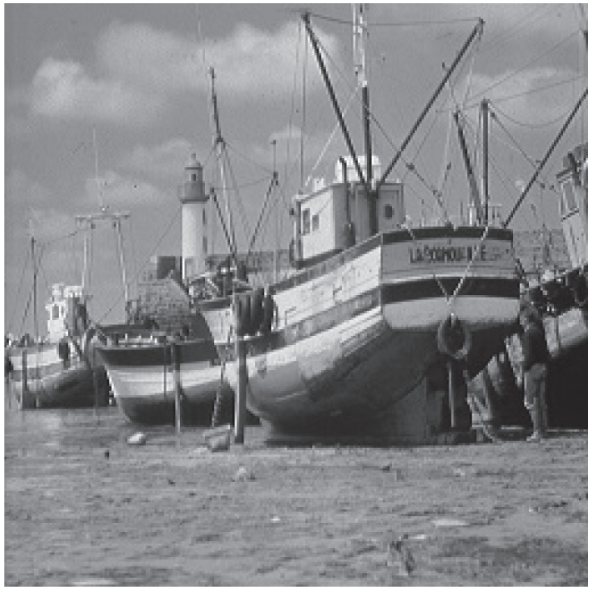

(d)

FIgURE 3: Continued. 


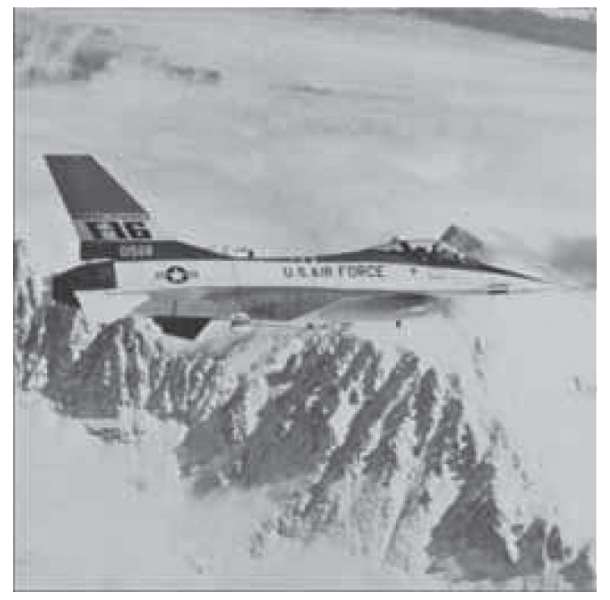

(e)

Figure 3: Standard test images used for evaluation. (a) Lena, (b) Baboon, (c) Peppers, (d) Boat, and (e) Airplane.

TABLE 4: PSNR for Lena, Peppers, and Boat using Airplane and Baboon as secret images.

\begin{tabular}{lccccc}
\hline \multirow{2}{*}{ Secret image } & \multicolumn{2}{c}{ Cover image } & Lena & Peppers \\
PSNR (dB) & Boat \\
PSNR (dB)
\end{tabular}

TABLE 5: Comparison between CPSO, PSO, and PSO-LSB-based methods.

\begin{tabular}{|c|c|c|c|c|c|c|}
\hline \multirow{2}{*}{ Stego-image } & \multicolumn{3}{|c|}{ PSNR } & \multicolumn{3}{|c|}{ SSIM } \\
\hline & PSO-LSB [11] & PSO [10] & CPSO & PSO-LSB [11] & PSO [10] & CPSO \\
\hline Baboon & 51.1487 & 57.84 & 63.253 & 0.9987 & 0.9990 & 0.9998 \\
\hline Lena & 51.1354 & 57.65 & 63.029 & 0.9982 & 0.9988 & 0.9996 \\
\hline Airplane & 51.1448 & 55.69 & 63.195 & 0.9955 & 0.9971 & 0.9995 \\
\hline Boat & 51.1413 & 55.89 & 63.109 & 0.9963 & 0.9965 & 0.9973 \\
\hline
\end{tabular}

to find the best pixel locations to embed the secret bits such that the PSNR of the stego-image is maximized.

To study the effect of the secret image size on the quality of the stego-image, Airplane and Baboon images with different resolutions are used as secret images and embedded into Lena, Peppers, and Baboon images of resolution $256 \times 256$; respectively. Table 4 shows the embedding capacity for different secret image sizes and the corresponding PSNR of the cover images. As shown, the PSNR is decreased when the embedding capacity increases; however, the corresponding PSNR values are still acceptable. For example, when the embedding capacity is 204,800 bits, the PSNR values for Lena are $56.145 \mathrm{~dB}$ and $60.1 \mathrm{~dB}$, respectively, which are still high.

The performance of the proposed method is compared with two related schemes: the PSO-based method in [10] and the PSO-LSB-based method in [11]. Table 5 shows the results of embedding Lena image with resolution $256 \times 256$ into different cover images (Baboon, Lena, Airplane, and Boat) of 

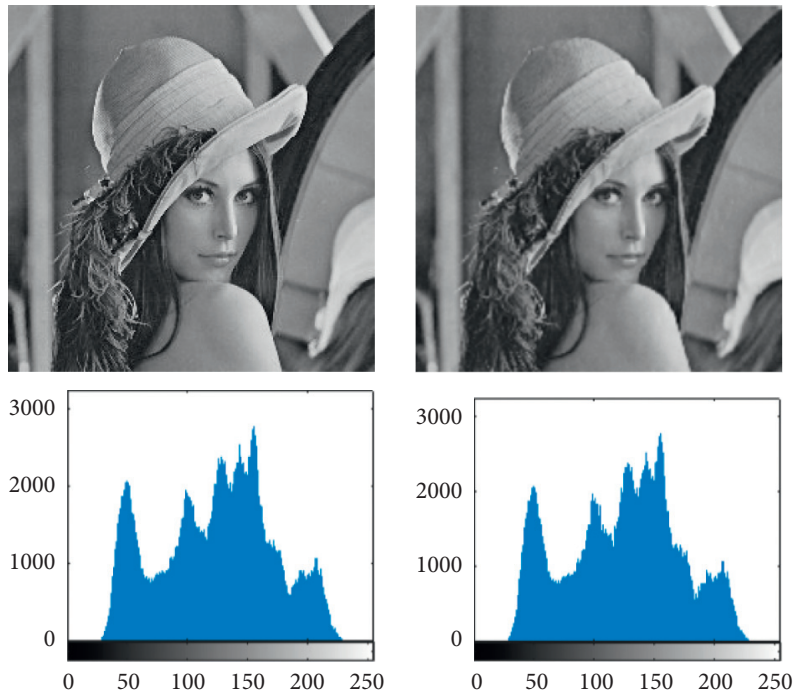

(a)
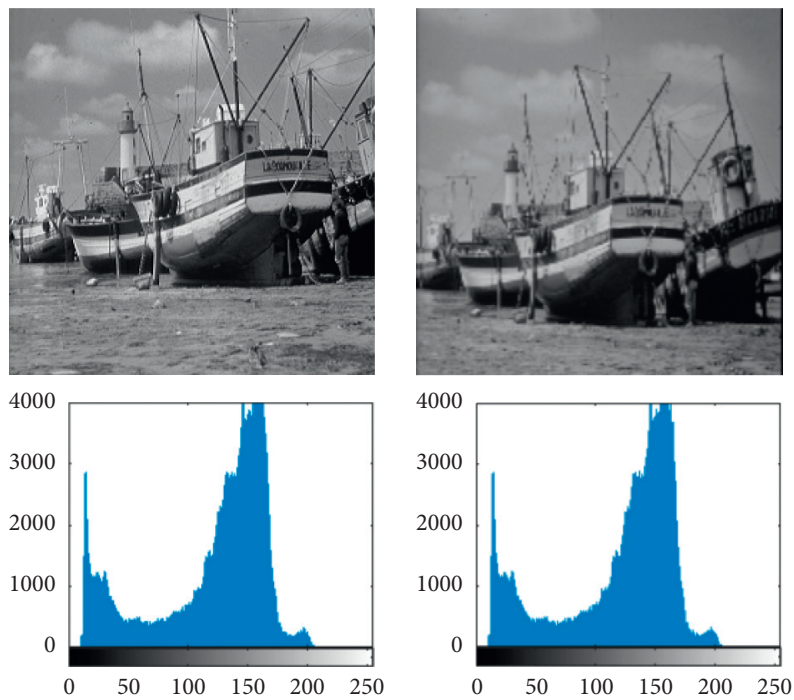

(c)
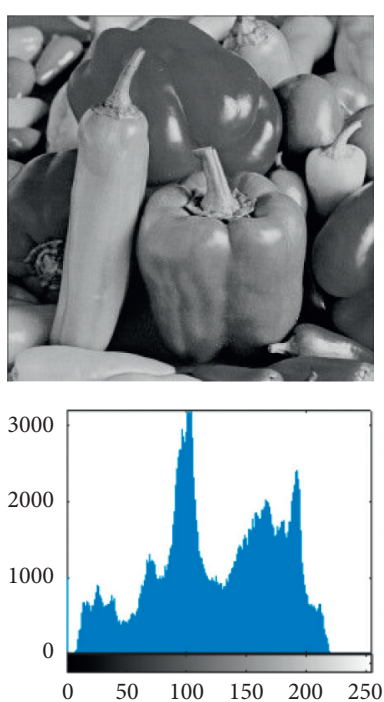
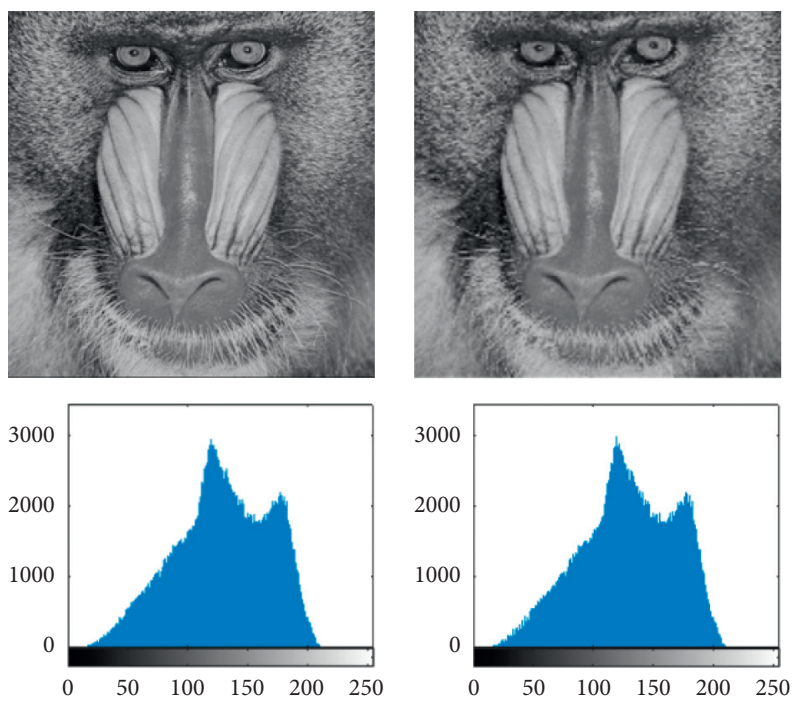

(b)
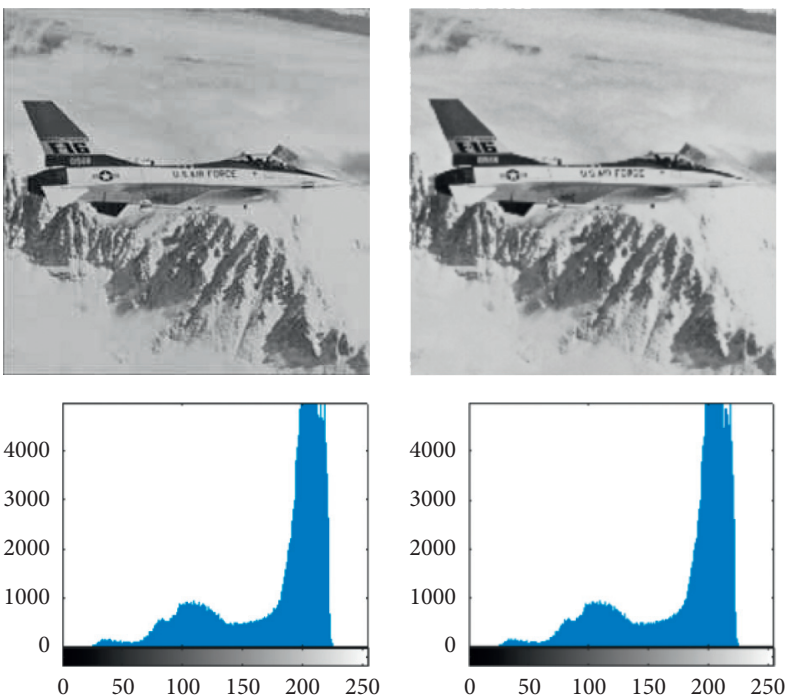

(d)
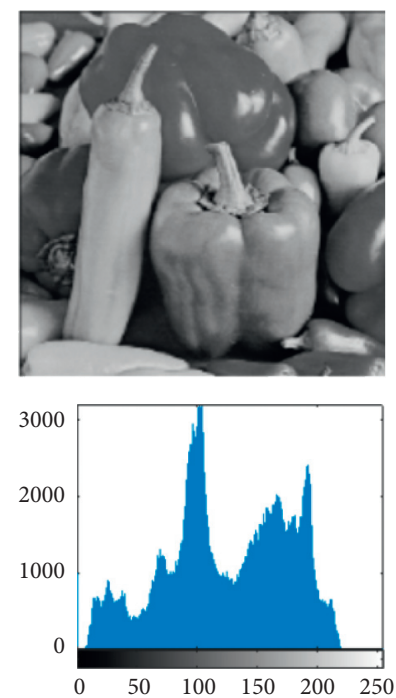

(e)

Figure 4: Cover and stego-images with their respective histograms. (a) Lena, (b) Baboon, (c) Boat, (d) Airplane, and (e) Peppers. 
resolution $512 \times 512$. For each method, the PSNR and SSIM of the stego-images are calculated. The results show the superiority of the proposed CPSO-based method over the other methods in terms of both PSNR and SSIM. For example, for the Lana image, the CPSO has PSNR $=63.029 \mathrm{~dB}$, while the PSO-LSB and PSO have $51.1354 \mathrm{~dB}$ and $57.65 \mathrm{~dB}$, respectively. In terms of SSIM, the CPSO has SSIM $=0.9996$, while the PSO-LSB and PSO have 0.9982 and 0.9988, respectively.

To further demonstrate the efficiency of the proposed method, histogram analysis is performed on the stegoimages in Table 5. Figure 4 shows the histograms of the cover and stego-images for Lena, Baboon, Airplane, and Boat. As shown, the cover and stego-images are visually alike and the histograms of the stego-images show no noticeable changes due to the minimal distortion of the stego pixels.

\section{Conclusion and Future Work}

We presented an efficient steganography method for concealing a secret image within a cover image based on chaotic maps and the PSO algorithm. The proposed CPSO algorithm is utilized to determine the best pixel locations in the cover image in order to embed the secret bits with minimal distortion. In the proposed method, we divide the cover and secret images into four blocks to improve the embedding capacity. The experimental results show that the proposed methods perform better than existing schemes in terms of PSNR and SSIM. As a future work, we plan to study other types of chaotic maps and investigate their effect on pixel selection in order to further enhance the efficiency of the CPSO algorithm.

\section{Data Availability}

The data are available on request to the corresponding author.

\section{Conflicts of Interest}

The authors declare that they have no conflicts of interest.

\section{References}

[1] B. B. Zaidan, A. A. Zaidan, and M. L. Mat Kiah, "Impact of data privacy and confidentiality on developing telemedicine applications: a review participates opinion and expert concerns," International Journal of Pharmacology, vol. 7, no. 3, pp. 382-387, 2011.

[2] A. K. Singh, J. Singh, and H. V. Singh, "Steganography in images using lsb technique," International Journal of Latest Trends in Engineering and Technology (IJLTET), vol. 5, no. 1, pp. 426-430, 2015.

[3] A. A. Zaidan, B. B. Zaidan, A. K. Al-Fraja, and H. A. Jalab, "Investigate the capability of applying hidden data in text file: an overview," Journal of Applied Sciences, vol. 10, no. 17, pp. 1916-1922, 2010.

[4] M. Hussain, A. W. A. Wahab, Y. I. B. Idris, A. T. S. Ho, and K.-H. Jung, "Image steganography in spatial domain: a survey," Signal Processing: Image Communication, vol. 65, pp. 46-66, 2018.

[5] B. B. Zaidan, A. A. Zaidan, A. K. Al-Frajat, and H. A. Jalab, "On the differences between hiding information and cryptography techniques: an overview," Journal of Applied Sciences, vol. 10, no. 15, pp. 1650-1655, 2010.

[6] I. J. Kadhim, P. Premaratne, P. J. Vial, and B. Halloran, "Comprehensive survey of image steganography: techniques, evaluations, and trends in future research," Neurocomputing, vol. 335, pp. 299-326, 2019.

[7] S. Kaur, A. Kaur, and K. Singh, "A survey of image steganography," IJRECE (International Journal of Research in Electronics and Computer Engineering), vol. 2, no. 3, pp. 102-105, 2014.

[8] D. Wang, D. Tan, and L. Liu, "Particle swarm optimization algorithm: an overview," Soft Computing, vol. 22, no. 2, pp. 387-408, 2018.

[9] D. Tian, "Particle swarm optimization with chaos-based initialization for numerical optimization," Intelligent Automation \& Soft Computing, vol. 24, no. 2, pp. 331-342, 2018.

[10] A. H. Mohsin, A. Zaidan, B. Zaidan et al., "New method of image steganography based on particle swarm optimization algorithm in spatial domain for high embedding capacity," IEEE Access, vol. 7, pp. 168994-169010, 2019.

[11] P. K. Muhuri, Z. Ashraf, and S. Goel, "A novel image steganographic method based on integer wavelet transformation and particle swarm optimization," Applied Soft Computing, vol. 92, Article ID 106257, 2020.

[12] H. A. Hefny and S. S. Azab, "Chaotic particle swarm optimization," in Proceedings of the 2010 7th International Conference on Informatics and Systems (INFOS), pp. 1-8, Cairo, Egypt, March 2010.

[13] Z. F. Yaseen and A. A. Kareem, "Image steganography based on hybrid edge detector to hide encrypted image using vernam algorithm," in Proceedings of the 2019 2nd Scientific Conference of Computer Sciences (SCCS), pp. 75-80, IEEE, Baghdad, Iraq, March 2019.

[14] Z. Li and Y. He, "Steganography with pixel-value differencing and modulus function based on pso," Journal of Information Security and Applications, vol. 43, pp. 47-52, 2018.

[15] A. K. Sahu and G. Swain, "Pixel overlapping image steganography using pvd and modulus function," $3 D$ Research, vol. 9, no. 3, p. 40, 2018.

[16] Z. Qu, Z. Li, G. Xu, S. Wu, and X. Wang, "Quantum image steganography protocol based on quantum image expansion and grover search algorithm," IEEE Access, vol. 7, pp. 50849-50857, 2019.

[17] P. D. Shah and R. S. Bichkar, "A secure spatial domain image steganography using genetic algorithm and linear congruential generator," in International Conference on Intelligent Computing and Applications, pp. 119-129, Springer, Berlin, Germany, 2018.

[18] R. Wazirali, W. Alasmary, M. M. Mahmoud, and A. Alhindi, "An optimized steganography hiding capacity and imperceptibly using genetic algorithms," IEEE Access, vol. 7, pp. 133496-133508, 2019.

[19] S. A. Nie, G. Sulong, R. Ali, and A. Abel, "The use of least significant bit (lsb) and knight tour algorithm for image steganography of cover image," International Journal of Electrical and Computer Engineering, vol. 9, no. 6, p. 5218, 2019.

[20] G. Swain, "Very high capacity image steganography technique using quotient value differencing and lsb substitution," 
Arabian Journal for Science and Engineering, vol. 44, no. 4, pp. 2995-3004, 2019.

[21] S. I. Nipanikar, V. Hima Deepthi, and N. Kulkarni, "A sparse representation based image steganography using particle swarm optimization and wavelet transform," Alexandria Engineering Journal, vol. 57, no. 4, pp. 2343-2356, 2018.

[22] S. Sajasi and A.-M. Eftekhari Moghadam, "An adaptive image steganographic scheme based on noise visibility function and an optimal chaotic based encryption method," Applied Soft Computing, vol. 30, pp. 375-389, 2015.

[23] P. S. Raja and E. Baburaj, "An efficient data embedding scheme for digital images based on particle swarm optimization with lsbmr," in Proceedings of the Third International Conference on Computational Intelligence and Information Technology (CIIT 2013), Mumbai, India, October 2013.

[24] S. Wang, B. Yang, and X. Niu, "A secure steganography method based on genetic algorithm," Journal of Information Hiding and Multimedia Signal Processing, vol. 1, no. 1, pp. 28-35, 2010.

[25] E. Ghasemi and J. Shanbehzadeh, "An imperceptible steganographic method based on genetic algorithm," in Proceedings of the 2010 5th International Symposium on Telecommunications, pp. 836-839, IEEE, Tehran, Iran, December 2010.

[26] T. Dongping, "Particle swarm optimization with chaotic maps and Gaussian mutation for function optimization," International Journal of Grid and Distributed Computing, vol. 8, no. 4, pp. 123-134, 2015.

[27] S. Rajendran and M. Doraipandian, "Chaotic map based random image steganography using lsb technique," International Journal of Network Security, vol. 19, no. 4, pp. 593598, 2017.

[28] Y. Yue, L. Cao, J. Hu, S. Cai, B. Hang, and H. Wu, "A novel hybrid location algorithm based on chaotic particle swarm optimization for mobile position estimation," IEEE Access, vol. 7, pp. 58541-58552, 2019.

[29] A. Singh and U. Jauhari, "A symmetric steganography with secret sharing and psnr analysis for image steganography," International Journal of Scientific and Engineering Research, vol. 3, no. 6, pp. 1279-1285, 2012.

[30] Z. Wang, A. C. Bovik, H. R. Sheikh, and E. P. Simoncelli, "Image quality assessment: from error visibility to structural similarity," IEEE Transactions on Image Processing, vol. 13, no. 4, pp. 600-612, 2004. 